\title{
Entanglement as a resource for local state discrimination in multipartite systems
}

\author{
Somshubhro Bandyopadhyay * and Saronath Halder \\ Department of Physics and Center for Astroparticle Physics and Space Science, \\ Bose Institute, Block EN, Sector V, Bidhan Nagar, Kolkata 700091 \\ Michael Nathanson ${ }^{\dagger}$ \\ Department of Mathematics and Computer Science, Saint Mary's College of California, Moraga, CA, 94556, USA
}

\begin{abstract}
We explore the question of using an entangled state as a universal resource for implementing quantum measurements by local operations and classical communication (LOCC). We show that for most systems consisting of three or more subsystems, there is no entangled state from the same space that can enable all measurements by LOCC. This is in direct contrast to the bipartite case, where a maximally entangled state is an universal resource. Our results are obtained showing an equivalence between the problem of local state transformation and that of entanglement-assisted local unambiguous state discrimination.
\end{abstract}

\section{INTRODUCTION}

Given a quantum system composed of spatiallyseparated subsystems, it is well known that the set of quantum operations which can be implemented with local operations and classical communication (LOCC) constitutes a strict subset of all quantum operations on the whole system. For example, it is impossible, by LOCC, to transform a product state into an entangled state [1], even with nonzero probability. The presence of additional entanglement, however, can help to overcome such restrictions [2-7]; and appropriate shared entanglement enables local implementation of any quantum operation. In this way, entanglement can be seen as a resource for quantum operations, e.g., quantum teleportation [8], superdense coding [9], entanglementcatalysis [10], entangling measurements and unitaries [2-6].

In this work, we explore the question of universal resource states, whose presence overcomes the limitations imposed by LOCC to allow implementation of whole classes of quantum operations. For instance, in a bipartite quantum system $\mathcal{H}=\mathcal{H}_{A} \otimes \mathcal{H}_{B}$, a maximally entangled state $|\Psi\rangle \in \mathcal{H}$ constitutes a universal resource for extracting classical information. This can be easily understood by imagining the subsystems as controlled by two parties, Alice and Bob, who share an unknown state $|\phi\rangle \in \mathcal{H}_{A} \otimes \mathcal{H}_{B}$. If they also share the maximally entangled state $|\Psi\rangle$, they can use it to teleport Alice's qudit to Bob. Bob can then extract information about $|\phi\rangle$ by applying any quantum measurement to his own system, thus overcoming the limitations of the spatial separation. Because Alice and Bob can use $|\Psi\rangle$ to perform any complete measurement on $|\phi\rangle$, we say that $|\Psi\rangle$ is universal for the task of complete measurement.

A natural question to ask is whether such universal resource states also exist for multipartite systems (those consisting of three or more subsystems), where entanglement has a more complex structure [11]. The present work considers the task of quantum state discrimination by LOCC [12-31] and seeks to characterize resource states which enable us to locally distinguish any set of orthogonal states from a fixed multipartite system. We show that for a given multipartite system, a universal resource state for local state discrimination must in general be from a larger-dimensional space than the states themselves. Moreover, there exist orthonormal bases that cannot be perfectly distinguished by LOCC using any resource state from the same size state space, making them good candidates for data hiding protocols. [32-35]

We prove our results by demonstrating an equivalence between local unambiguous state discrimination and local state transformation. Any LOCC protocol which implements one task can be adapted to achieve the other. Since multipartite entanglement is understood primarily in terms of local state transformation, our equivalence gives us tools to understand both unambiguous and perfect discrimination with LOCC. This equivalence (which follows similar results in [24] and others) is useful in its own right and nicely complements recent results on resources for local state transformation [36]. This work extends earlier analysis of entanglement as a resource in bipartite systems, which often calculate how much additional entanglement is necessary to complete a certain task locally. (Early examples include quantum teleportation and entanglement catalysis for state discrimination [1? ? ].) Given the complexity of quantifying the amount of multipartite entanglement necessary for a given task, we must pay attention to the quality of the entanglement instead.

The rest of the paper explores these questions in the following way. In Section [I], we give a precise definition of a universal resource and full statements of our results, which are proven in Section III] In Section IV, we illustrate our results with examples, while Section $\nabla$ gives conclusions and directions for future work. 


\section{STATEMENT OF RESULTS}

\section{A. Entanglement as a Resource for LOCC Measurement}

Local state discrimination problems assume that classical information has been encoded in quantum states and seek to determine how much of this information can be recovered locally. Formally, we suppose that a set of spatially separated observers share a quantum system prepared in one of a known set of pure states $\left\{\left|\psi_{i}\right\rangle\right\} \subset \otimes_{k=1}^{N} \mathcal{H}_{k}$, each occurring with some nonzero probability; see e.g., [12-20]. The question of interest is, can LOCC protocols distinguish such quantum states as well as global measurements can?

Although optimal discrimination of two states is always possible to implement using only LOCC [12, 31], local discrimination of larger sets in general is not [14, 26]. In particular, if $\mathcal{B}$ is a complete basis consisting of multipartite states, LOCC is never sufficient to distinguish its elements if $\mathcal{B}$ contains any entangled states [24]. It may be noted that the existence of locally indistinguishable states imply locally hidden information, and has thus found applications in quantum cryptography primitives such as data hiding and secret sharing [32-35, 37].

On the other hand, sets of locally indistinguishable quantum states may become distinguishable in the presence of shared entanglement, e.g., [7, 30], and this is the phenomenon we wish to understand better. Imagine that Alice and Bob are initially in the same location prior to going their separate ways. They know that in the future, they will need to distinguish a set of bipartite states from $\mathcal{H} \otimes \mathcal{H}$; but they do not know what these states will be. Nonetheless, if they prepare a maximally entangled state $|\Psi\rangle \in \mathcal{H}_{A} \otimes \mathcal{H}_{B}$, then for any set of states $\left\{\left|\psi_{i}\right\rangle\right\} \subset \mathcal{H}_{A} \otimes \mathcal{H}_{B}$, the states $\left\{|\Psi\rangle \otimes\left|\psi_{i}\right\rangle\right\}$ can be distinguished as well using only LOCC across the $A: B$ split as they can be using any other quantum operations. The maximally entangled state is a true resource in that it enables measurements which are not otherwise possible but is consumed in the process. And it is universalAlice and Bob can prepare $|\Psi\rangle$ without knowing what the $\left\{\left|\psi_{i}\right\rangle\right\}$ will be.

In this work, we seek to explore this same idea in the context of multipartite systems. If Alice, Bob, and Charlie wish to jointly prepare an entangled resource state to help them distinguish unknown sets from $\mathcal{H} \otimes \mathcal{H} \otimes \mathcal{H}$, it is less clear what this resource state should be; and in fact depends on the type of state discrimination they are trying to achieve. This motivates the primary definition in this work.

Definition 1. Let $\mathcal{H}=\otimes_{k=1}^{N} \mathbb{C}^{d_{k}}$ be a multipartite system and let $\mathcal{H}^{\prime}=\otimes_{k=1}^{N} \mathbb{C}^{d_{k}^{\prime}}$ be a system with the same multipartite structure as $\mathcal{H}$. Consider $\mathcal{H}^{\prime} \otimes \mathcal{H}$ as an $N$-partite system with local subsystems $\left(\mathbb{C}^{d_{k}^{\prime}} \otimes \mathbb{C}^{d_{k}}\right), k=1, \ldots, N$.

We say that a state $|\Phi\rangle \in \mathcal{H}^{\prime}$ is universal for local state discrimination in $\mathcal{H}$ if, for every set $\mathcal{B}=\left\{\left|\psi_{i}\right\rangle\right\}$ of $m u$ tually orthogonal states in $\mathcal{H}$, the set of states $|\Phi\rangle \otimes \mathcal{B} \equiv$ $\left\{|\Phi\rangle \otimes\left|\psi_{i}\right\rangle\right\} \subset \mathcal{H}^{\prime} \otimes \mathcal{H}$ can be perfectly distinguished with LOCC.

Likewise, $|\Phi\rangle$ is universal for local unambiguous discrimination in $\mathcal{H}$ if, for every set $\mathcal{B}=\left\{\left|\psi_{i}\right\rangle\right\}$ of linearly independent states in $\mathcal{H}$, the set of states $|\Phi\rangle \otimes \mathcal{B} \equiv$ $\left\{|\Phi\rangle \otimes\left|\psi_{i}\right\rangle\right\} \subset \mathcal{H}^{\prime} \otimes \mathcal{H}$ can be unambiguously distinguished with LOCC.

We insist that our states be mutually orthogonal for perfect state discrimination, as this is the necessary and sufficient condition for them to be perfectly distinguishable using full quantum operations. Likewise, we assume linear independence for unambiguous state discrimination (defined in the next section), since this is the necessary and sufficient condition when we are not limited by locality. [38]

A maximally entangled bipartite state constitutes a universal resource for both local state discrimination and local unambiguous discrimination. In order to explore the analogous question in multipartite systems, we need to develop necessary conditions for resource states relative to each task.

\section{B. Local state transformations}

Our first result gives a strong equivalence between local state transformation and the problem of unambiguous state discrimination. In unambiguous state discrimination, definitive knowledge of the state is balanced against a probability of definitive failure. That is, given a state $|\psi\rangle \in\left\{\left|\psi_{i}\right\rangle\right\}$, we either conclude with certainty that $|\psi\rangle=\left|\psi_{i}\right\rangle$ or else we receive a failure indication. As long as each $\left|\psi_{i}\right\rangle$ is detectable with positive probability, we say that the set of states can be unambiguously discriminated; it is not hard to show that this condition is equivalent to the linear independence of the $\left|\psi_{i}\right\rangle$. [38]

If we are restricted to local operations and classical communications, it can be useful to employ entanglement in the form of a resource state $|\Phi\rangle$. We say that the set of states $\left\{|\Phi\rangle \otimes\left|\psi_{i}\right\rangle, 1 \leq i \leq D\right\} \in \mathcal{H}^{\prime} \otimes \mathcal{H}$ is unambiguously locally distinguishable if there exists an LOCC measurement $\Pi=\left\{\Pi_{i}\right\}_{i=1}^{D+1}$ satisfying $\sum_{i} \Pi_{i}=\mathcal{I}_{\mathcal{H}^{\prime} \otimes \mathcal{H}}$ such that for all $i, j \in\{1,2, \ldots D\}$,

$$
\left\langle\Phi \otimes \psi_{j}\left|\Pi_{i}\right| \Phi \otimes \psi_{j}\right\rangle=\delta_{i j} \epsilon_{i}
$$

where $\delta_{i j}$ is the Kronecker delta and the $\epsilon_{i}$ are positive constants. The outcome $(D+1)$ is inconclusive. It turns out that the task of using entanglement to enable local unambiguous discrimination is closely related to that of transforming one state into another using only LOCC. 
Theorem 1. Let $\mathcal{B}=\left\{\left|\psi_{i}\right\rangle\right\}$ be a complete basis of $\mathcal{H}=$ $\otimes_{k=1}^{N} \mathbb{C}^{d_{k}}$, not necessarily orthogonal. For each $i$, define $\left|\tilde{\psi}_{i}\right\rangle$ to be the unique state in $\mathcal{H}$ which is orthogonal to every $\left|\psi_{j}\right\rangle$ with $j \neq i$.

Let $|\Phi\rangle$ be a pure state in $\mathcal{H}^{\prime}=\otimes_{k=1}^{N} \mathbb{C}^{d_{k}^{\prime}}$; and denote by $\left|\Phi^{*}\right\rangle$ the entrywise complex conjugate of $|\Phi\rangle$ taken in the standard basis. Then the set of states $|\Phi\rangle \otimes \mathcal{B} \equiv$ $\left\{|\Phi\rangle \otimes\left|\psi_{i}\right\rangle\right\} \in \mathcal{H}^{\prime} \otimes \mathcal{H}$ can be unambiguously distinguished by LOCC if and only if there exists an LOCC protocol which transforms $\left|\Phi^{*}\right\rangle$ into each $\left|\tilde{\psi}_{i}\right\rangle$ with positive probability.

In particular, if we can use LOCC to unambiguously identify each $\left|\psi_{i}\right\rangle$ with probability $\epsilon_{i}>0$, then there exists an LOCC protocol which transforms $\left|\Phi^{*}\right\rangle$ into each $\left|\tilde{\psi}_{i}\right\rangle$ with probability at least $\frac{\epsilon_{i}}{D}$.

This theorem is proved in Section [II by directly showing how a local unambiguous measurement can be used to build a local transformation protocol, and vice versa. As an immediate consequence, we get a necessary condition for distinguishing orthonormal bases, where for each $i,|\tilde{\psi}\rangle=|\psi\rangle$.

Corollary 1. Let $\mathcal{B}=\left\{\left|\psi_{i}\right\rangle\right\} \subset \mathcal{H}=\otimes_{i=1}^{N} \mathbb{C}^{d_{i}}$ be a complete orthonormal basis of $\mathcal{H}$, and let $|\Phi\rangle \in \mathcal{H}^{\prime}=\otimes_{i=1}^{N} \mathbb{C}^{d_{i}^{\prime}}$ be fixed resource state (with $d_{i}^{\prime}$ not necessarily equal to $\vec{d}_{i}$ ).

If the set of states $|\Phi\rangle \otimes \mathcal{B} \equiv\left\{|\Phi\rangle \otimes\left|\psi_{i}\right\rangle\right\} \in \mathcal{H}^{\prime} \otimes \mathcal{H}$ can be perfectly distinguished by LOCC then there exists a LOCC protocol by means of which $\left|\Phi^{*}\right\rangle$ is converted to each of the $\left|\psi_{i}\right\rangle$ with probability $p=\frac{1}{D}$.

Corollary 1 follows immediately from the last piece of Theorem 1, setting each $\epsilon_{i}=1$ and observing that if each $p_{i} \geq \frac{1}{D}$, then this must be an equality in order for the sum of the $p_{i}$ to equal one. Note that if we let $|\Phi\rangle$ be a product state, then Theorem 1 implies Theorem 3 of [21], which states that, without additional entanglement, a basis $\mathcal{B}$ is unambiguously distinguishable with LOCC iff each $\left|\tilde{\psi}_{i}\right\rangle$ is a product state. Similarly, Corollary 1 implies the fundamental result of [24] that a complete basis can be locally distinguished only if it contains only product states.

\section{Entanglement classes and universal resources}

We return now to the question for finding universal resources for local state discrimination. The challenge in finding a universal resource is a consequence of the complex structure of multipartite entanglement, which is often described in terms of stochastic LOCC equivalence classes. Two states $\left|\psi_{1}\right\rangle$ and $\left|\psi_{2}\right\rangle$ are considered to be in the same SLOCC class $\mathcal{C}$ if it is possible to use LOCC to transform the state $\left|\psi_{1}\right\rangle$ into $\left|\psi_{2}\right\rangle$ with a positive probability of success, and also to effect the transformation $\left|\psi_{2}\right\rangle \rightarrow\left|\psi_{1}\right\rangle$. The SLOCC equivalence classes form a partially ordered set, with $\mathcal{C}_{1} \preceq \mathcal{C}_{2}$ if the transformation $\left|\phi_{2}\right\rangle \rightarrow\left|\phi_{1}\right\rangle$ is possible with LOCC for $\left|\phi_{i}\right\rangle \in \mathcal{C}_{i}$.

For a given bipartite system $\mathcal{H}=\mathcal{H}_{A} \otimes \mathcal{H}_{B}$, the SLOCC partial ordering is characterized by the existence of least upper bounds. Even though there are pairs of states which are locally incompatible $\left(\left|\phi_{1}\right\rangle \not \rightarrow\left|\phi_{2}\right\rangle\right.$ and $\left.\left|\phi_{2}\right\rangle \not \rightarrow\left|\phi_{1}\right\rangle\right)$ when $d>2$, there always exists a unique SLOCC class $\mathcal{C}_{\text {max }}$ of maximally-entangled states such that if $|\phi\rangle \in \mathcal{C}_{\max }$, then the local transformation $|\phi\rangle \rightarrow|\psi\rangle$ is possible for all $|\psi\rangle \in \mathcal{H}$ (and is, in fact, possible with probability 1 ).

An SLOCC class of $\mathcal{H}$ is maximal if it is not reached from any other SLOCC class of $\mathcal{H}$. Equivalently, we say that $\mathcal{C}$ is maximal if, for all $|\phi\rangle \in \mathcal{C}$ and $|\psi\rangle \in \mathcal{H}$, $|\psi\rangle \rightarrow|\phi\rangle$ implies that $|\psi\rangle \in \mathcal{C}$. We refer to states in maximal SLOCC classes as maximally entangled. Most multipartite systems have more than one maximally entangled equivalence class [11, 39]; combined with Theorems 1 and Corollary 1, this has consequences in our search for a universal resource:

Theorem 2. Consider an N-partite system $\mathcal{H}=\otimes_{k=1}^{N} \mathbb{C}^{d_{k}}$ with $N \geq 3, d_{k} \geq 2$ for all $k$. If $\mathcal{H}$ contains more than one maximally entangled equivalence class, then there does not exist a universal resource state $|\Phi\rangle \in \mathcal{H}$ for either local state discrimination or local unambiguous discrimination.

In fact, there exist orthonormal bases $\mathcal{B}$ of $\mathcal{H}$ for which the set of states $|\Phi\rangle \otimes \mathcal{B}$ is not locally distinguishable for any $|\Phi\rangle \in \mathcal{H}$.

Corollary 2. For any $\mathcal{H}=\left(\mathbb{C}^{d}\right)^{\otimes N}$ with $N \geq 3, d \geq 2$, there does not exist a universal resource state $|\Phi\rangle \in \mathcal{H}$ for either local state discrimination or local unambiguous discrimination.

The assumption in Theorem 2 that $\mathcal{H}$ contains a pair of incompatible maximally entangled states is typical for multipartite spaces. For instance, in the case of three qubits, the GHZ states and the $W$ states are both maximally entangled but cannot be transformed into each other, so there is no three-qubit pure state that can optimally distinguish all three-qubit orthonormal bases. Any universal resource state must exist in higher dimensions.

The second part of the theorem says that it may not be possible to find even a basis-dependent resource state from the same state space, i.e., there exists sets of multipartite states for which no multipartite pure state from the same state space can perfectly distinguish them by LOCC. Again in the case of three qubits, an example would be any basis which contains at least one GHZ state and one $W$ state (see [11] and equation (5)). For such a basis there is no three-qubit resource state which will enable perfect state discrimination. Clearly, in this case any resource state that perfectly distinguishes the basis using LOCC requires higher dimensions as well. 
To see the connection between Theorems 1 and 2, we assume that $\mathcal{H}$ is a multipartite space with two distinct maximal SLOCC classes $\mathcal{C}_{1}$ and $\mathcal{C}_{2}$ with pure states $\left|\psi_{1}\right\rangle \in \mathcal{C}_{1}$ and $\left|\psi_{2}\right\rangle \in \mathcal{C}_{2}$. Since these classes are maximal, we know that if $|\Phi\rangle \in \mathcal{H}$ and $|\Phi\rangle \rightarrow\left|\psi_{i}\right\rangle$, then $|\Phi\rangle \in \mathcal{C}_{i}$ for $i=1,2$. Since equivalence classes must be disjoint, there does not exist a state $|\Phi\rangle \in \mathcal{H}$ which can be transformed into both $\left|\psi_{1}\right\rangle$ and $\left|\psi_{2}\right\rangle$. By Corollary 1 , if $\mathcal{B}_{1}$ and $\mathcal{B}_{2}$ are orthonormal bases for $\mathcal{H}$ with $\left|\psi_{i}\right\rangle \in \mathcal{B}_{i}$, then it is not possible that the sets $|\Phi\rangle \otimes \mathcal{B}_{1}$ and $|\Phi\rangle \otimes \mathcal{B}_{2}$ are both perfectly (or even unambiguously) distinguishable with LOCC. Since this is true for any $|\Phi\rangle \in \mathcal{H}$, then there is no universal resource state in $\mathcal{H}$.

Note also if we look at a basis $\mathcal{B}$ which contains both $\left|\psi_{1}\right\rangle$ and $\left|\psi_{2}\right\rangle$ and apply Theorem 1 , then the set $|\Phi\rangle \otimes \mathcal{B}$ cannot be unambiguously distinguished for any $|\Phi\rangle \in$ $\mathcal{H}$, even if we allow $|\Phi\rangle$ to depend on $\mathcal{B}$.

As was previously observed, if we allow $|\Phi\rangle$ to live in a higher-dimensional space, then it might be possible to convert into different maximal SLOCC classes of $\mathcal{H}$. While characterizing universal resource states for perfect discrimination has proved challenging, our results give necessary and sufficient conditions for unambiguous discrimination as a corollary to Theorem 1

Corollary 3. Let $\mathcal{H}=\otimes_{k=1}^{N} \mathbb{C}^{d_{k}}$ be a multipartite system and let $\mathcal{H}^{\prime}=\otimes_{k=1}^{N} \mathbb{C}^{d_{k}^{\prime}}$ be a system with the same multipartite structure as $\mathcal{H}$.

Then $|\Phi\rangle \in \mathcal{H}^{\prime}$ is a universal resource for unambiguous discrimination if and only if for every maximally entangled state $|\phi\rangle \in \mathcal{H},\left|\Phi^{*}\right\rangle$ can be locally transformed into $|\phi\rangle$.

That is, to test whether a state is universal for unambiguous discrimination in $\mathcal{H}$, one need only test whether it can be transformed into each of the maximallyentangled states of $\mathcal{H}$. (Characterizing the set of maximally-entangled states was the focus of recent work in [41].) The corollary follows since, for every $\left|\phi^{\prime}\right\rangle \in \mathcal{H}$, there exists a maximally entangled state $|\phi\rangle$ which can be locally transformed into $\left|\phi^{\prime}\right\rangle$. Hence, $\left|\Phi^{*}\right\rangle \rightarrow|\phi\rangle \rightarrow\left|\phi^{\prime}\right\rangle$, and we can apply Theorem 1

\section{PROOF OF THEOREM 1}

We begin by showing that local unambiguous discrimination implies local transformation with fixed probabilities of success. The following lemma is easily checked:

Lemma 1. Let $\left\{\left|\psi_{i}\right\rangle\right\}$ be a complete basis of $\mathcal{H}$, not necessarily orthogonal; and for each $i$, let $\left|\tilde{\psi}_{i}\right\rangle$ be the unique unit vector in $\mathcal{H}$ such that $\left\langle\tilde{\psi}_{i} \mid \psi_{j}\right\rangle=0$ if $i \neq j$.

If $\operatorname{dim} D=\mathcal{H}, \mathcal{I}$ is the identity operator on $\mathcal{H}$ and $|\Psi\rangle$ is the standard maximally-entangled state on $\mathcal{H} \otimes \mathcal{H}$, then we can write

$$
\mathcal{I}=\sum_{i} \frac{1}{\left\langle\tilde{\psi}_{i} \mid \psi_{i}\right\rangle}\left|\psi_{i}\right\rangle\left\langle\tilde{\psi}_{i}\right|
$$

and

$$
|\Psi\rangle=\frac{1}{\sqrt{D}} \sum_{i} \frac{1}{\left\langle\tilde{\psi}_{i} \mid \psi_{i}\right\rangle}\left|\psi_{i}^{*}\right\rangle\left|\tilde{\psi}_{i}\right\rangle
$$

where $\left|\psi_{i}^{*}\right\rangle$ is the entrywise complex conjugate of $\left|\psi_{i}\right\rangle$.

Now suppose that there exists an LOCC measurement $\Pi=\left\{\Pi_{i}\right\}_{i=1}^{D}$ satisfying $\sum_{i} \Pi_{i}=\mathcal{I}_{\mathcal{H}^{\prime} \otimes \mathcal{H}}$ such that

$$
\left\langle\Phi \otimes \psi_{j}\left|\Pi_{i}\right| \Phi \otimes \psi_{j}\right\rangle=\delta_{i, j} \epsilon_{i}
$$

for $\epsilon_{i}>0$. We show that there exists a local protocol which effects the transformation $\left|\Phi^{*}\right\rangle \rightarrow\left|\psi_{i}\right\rangle$ with probability $\frac{\epsilon_{i}}{D}$ for each $i$.

Suppose that $N$ spatially-separated observers $\left\{O_{k}\right\}$ each control a subsystem of $\mathcal{H}^{\prime}$ and initially share the state $\left|\Phi^{*}\right\rangle \in \mathcal{H}^{\prime}$. Each observer $O_{k}$ locally produces the maximally entangled state $\left|\Psi_{k}\right\rangle \in \mathbb{C}^{d_{k}} \otimes \mathbb{C}^{d_{k}}$ in an ancillary system. We can then write the maximally entangled state on $\mathcal{H} \otimes \mathcal{H}$ as $|\Psi\rangle=\otimes_{k=1}^{N}\left|\Psi_{k}\right\rangle$. This means that the state of our entire system is given by

$$
\left|\Phi^{*}\right\rangle \otimes|\Psi\rangle \in \mathcal{H}^{\prime} \otimes(\mathcal{H} \otimes \mathcal{H})
$$

Using lemma1, we can write

$$
\begin{aligned}
|\Psi\rangle & =\frac{1}{\sqrt{D}} \sum_{i} \frac{1}{\left\langle\tilde{\psi}_{i} \mid \psi_{i}\right\rangle} \otimes\left|\psi_{i}^{*}\right\rangle\left|\tilde{\psi}_{i}\right\rangle \\
\left|\Phi^{*}\right\rangle \otimes|\Psi\rangle & =\frac{1}{\sqrt{D}} \sum_{i} \frac{1}{\left\langle\tilde{\psi}_{i} \mid \psi_{i}\right\rangle}\left|\Phi^{*}\right\rangle \otimes\left|\psi_{i}^{*}\right\rangle \otimes\left|\tilde{\psi}_{i}\right\rangle
\end{aligned}
$$

Notice that we have created our copy of $|\Psi\rangle$ using only local operations on our $N$ subsystems. Now, we take the entrywise conjugate of each operator of our LOCC measurement $\Pi$ and apply the new measurement $\Pi^{*}$ to the first two systems. If we get the outcome $x$, then our system has been transformed as

$$
\begin{aligned}
\left(\sqrt{\Pi_{x}^{*}} \otimes \mathcal{I}\right)\left|\Phi^{*}\right\rangle|\Psi\rangle & =\frac{1}{\sqrt{D}} \sum_{i} \sqrt{\Pi_{x}^{*}}\left(\left|\Phi^{*}\right\rangle\left|\psi_{i}^{*}\right\rangle\right) \otimes\left|\psi_{i}\right\rangle \\
& =\frac{1}{\sqrt{D}} \sqrt{\Pi_{x}^{*}}\left(\left|\Phi^{*}\right\rangle\left|\psi_{x}^{*}\right\rangle\right) \otimes\left|\psi_{x}\right\rangle
\end{aligned}
$$

Tracing out the first two systems shows that our last system ends up in the state $\left|\psi_{x}\right\rangle$ with probability $\frac{1}{D}\left\langle\Phi \otimes \psi_{x}\left|\Pi_{x}\right| \Phi \otimes \psi_{x}\right\rangle=\frac{\epsilon_{x}}{D}>0$, which was to be shown.

To prove the converse, we assume that the LOCC transformation $\left|\Phi^{*}\right\rangle \rightarrow\left|\tilde{\psi}_{i}\right\rangle$ is possible for each $i$. This implies that there exists a product matrix $M_{i}=$ $\otimes_{k=1}^{N} M_{i}^{(k)}$ with $\operatorname{Tr} M_{i}^{*} M_{i}=D^{\prime}$ and $\left|\tilde{\psi}_{i}\right\rangle=\mu_{i} M_{i}\left|\Phi^{*}\right\rangle$ for 
some constant $\mu_{i}$. We will use this to build a measurement which unambiguously distinguishes the $\left\{\left|\psi_{i}\right\rangle\right\}$.

For each subsystem $k$, let $\left|\Psi_{k}^{\prime}\right\rangle$ be the standard maximally-entangled $d_{k}^{\prime} \otimes d_{k}^{\prime}$ state so that $\left|\Psi^{\prime}\right\rangle=$ $\otimes_{k=1}^{N}\left|\Psi_{k}^{\prime}\right\rangle$ is maximally entangled on $\mathcal{H}^{\prime} \otimes \mathcal{H}^{\prime}$. For each $i$, we can also define $\left|\phi_{i}\right\rangle=\left(I \otimes M_{i}\right)\left|\Psi^{\prime}\right\rangle$, which is a product state across our $N$ subsystems. This allows us to write

$$
\begin{aligned}
\left|\phi_{i}\right\rangle & =\otimes_{k=1}^{N}\left(I \otimes M_{i}^{(k)}\left|\Psi_{k}^{\prime}\right\rangle\right) \\
& =\left(I \otimes M_{i}\right)\left|\Psi^{\prime}\right\rangle \\
\left\langle\left(\Phi \otimes \psi_{j}\right) \mid \phi_{i}\right\rangle & =\left\langle\left(\Phi \otimes \psi_{j}\right)\left|\left(I \otimes M_{i}\right)\right| \Psi^{\prime}\right\rangle \\
& =\frac{1}{\sqrt{D^{\prime}}}\left\langle\psi_{j}\left|M_{i}\right| \Phi^{*}\right\rangle=\frac{1}{\sqrt{D^{\prime}}}\left\langle\psi_{j} \mid \tilde{\psi}_{i}\right\rangle
\end{aligned}
$$

By definition, $\left\langle\left(\Phi \otimes \psi_{j}\right) \mid \phi_{i}\right\rangle=\left\langle\psi_{j} \mid \tilde{\psi}_{i}\right\rangle \neq 0$ if and only if $i=j$. Since the $\left\{\left|\phi_{i}\right\rangle\right\}$ are product states, Lemma 3 [21] establishes that they can be used to unambiguously distinguish the states $\left\{\left|\Phi \otimes \psi_{i}\right\rangle\right\}$ using only LOCC. QED

\section{EXAMPLES}

When we look for universal resources in multipartite systems, we can mimic the bipartite structure to identify an example of a universal resource state: If one of the subsystems shares sufficient bipartite entanglement with each of the other subsystems, then teleportation can be used to recreate the entire unknown state in one location, after which discrimination is possible.

Example 1. Let $\mathcal{H}=\otimes_{k=1}^{N} \mathbb{C}^{d_{k}}$ be a multipartite system with $D=\operatorname{dim} \mathcal{H}=d_{1} d_{2} \cdots d_{N}$. We define a state in which each of the first $(N-1)$ subsystems shares a maximally-entangled state with the Nth system:

$$
\left|\Phi_{\text {Bell }}^{N}\right\rangle:=\sqrt{\frac{d_{N}}{D}} \sum_{i=1}^{D / d_{N}}|i \otimes i\rangle \in\left(\otimes_{k=1}^{N-1} \mathbb{C}^{d_{k}}\right) \otimes \mathbb{C}^{D / d_{N}}
$$

Then $\left|\Phi_{\text {Bell }}^{N}\right\rangle$ is a universal resource for both local unambigious discrimination and local perfect discrimination in $\mathcal{H}$.

Note that in general this requires the resource state to exist in a much higher-dimensional system that our original states, since the dimension of the last subsystem is the product of the dimensions of all the other subsystems.

This is not the only possible resource state, however. Corollary 3 implies that $|\Phi\rangle$ is a universal resource for unambiguous discrimination if and only if its SLOCC class is an upper bound for every SLOCC class in $\mathcal{H}$. This requires $|\Phi\rangle$ to possess sufficient entanglement, as measured by any entanglement monotone. In particular, we can look at the Schmidt measure

$$
E_{S}(|\Phi\rangle):=\log r
$$

where $r$ is the minimum number of terms in any representation of $|\Phi\rangle$ as a linear combination of product states. In bipartite systems, $r$ is simply the rank of the reduced density matrix, but $E_{S}$ is well-defined as an entanglement monotone in multipartite systems as well [11, 42-44]. The Schmidt rank of a pure state cannot increase under LOCC, which implies that for any universal resource state $|\Phi\rangle, E_{S}(|\Phi\rangle) \geq E_{S}(|\psi\rangle)$ for all $|\psi\rangle \in \mathcal{H}$. This necessary condition leads us to a second class of universal resource states for unambiguous discrimination:

Example 2. Let $\mathcal{H}=\otimes_{k=1}^{N} \mathbb{C}^{d_{k}}$ be a multipartite system. Following the notation in [45], we define $\left|G H Z_{N}^{R}\right\rangle \in$ $\left(\mathbb{C}^{R}\right)^{\otimes N}$ to be the generalized $G H Z$ state in dimension $R$ with $N$ parties:

$$
\left|G H Z_{N}^{R}\right\rangle=\frac{1}{\sqrt{R}} \sum_{i=0}^{(R-1)}|i\rangle^{\otimes N}
$$

Then $\left|G H Z_{N}^{R}\right\rangle$ is a universal resource for unambiguous discrimination in $\mathcal{H}$ if and only if

$$
\log R \geq \max _{|\psi\rangle \in \mathcal{H}} E_{S}(|\psi\rangle)
$$

The necessity follows from the monotonicity of the Schmidt measure. The sufficiency is given as Observation 1 in [45], which establishes that this state can be transformed into any state with smaller Schmidt rank; and the specific case when $\mathcal{H}=\left(\mathbb{C}^{2}\right)^{\otimes 3}$ is proved in [36]. The proof is immediate: We can write any state $|\psi\rangle \in \mathcal{H}$ as a linear combination of at most $R$ product states:

$$
|\psi\rangle=\sum_{i=1}^{R} \alpha_{i} \otimes_{k=1}^{N}\left|a_{i, k}\right\rangle
$$

If we define $A_{k}=\sum_{i}\left(\alpha_{i}\right)^{1 / N}\left|a_{i, k}\right\rangle\langle i|$, then we can write

$$
|\psi\rangle=\sqrt{R}\left(\otimes_{k=1}^{N} A_{k}\right)\left|G H Z_{N}^{R}\right\rangle
$$

which shows that we can locally transform $\left|G H Z_{N}^{R}\right\rangle$ into any state $|\psi\rangle \in \mathcal{H}$.

The maximum rank $R$ of $\mathcal{H}$ defined in this example is not easily calculated. However, the example of generalized $W$ states in [45, 46] give us that if $d=2^{n}$ and $\mathcal{H}=\left(\mathbb{C}^{d}\right)^{\otimes N}$, then $R \geq(N-1)(d-1)+1$.

This now gives us two classes of universal resources which are opposite extremes: $\left|\Phi_{\text {Bell }}^{N}\right\rangle$ has minimal dimension in all parties except one, while $\left|G H Z_{N}^{R}\right\rangle$ has uniform dimensions across each party and the minimum dimension for which this is possible. For instance, in the case of three qubits, the minimum rank is $R=3$, so $\left|G H Z_{N}^{R}\right\rangle$ lives in a $3 \otimes 3 \otimes 3$ system, while $\left|\Phi_{\text {Bell }}^{N}\right\rangle$ lives 
in a $4 \otimes 2 \otimes 2$ system. It is an open question to characterize all universal resource states for a fixed system $\mathcal{H}$.

Note that $\left|\Phi_{\text {Bell }}^{N}\right\rangle$ is clearly a universal state for perfect discrimination as well as unambiguous discrimination, while it is not clear whether this is true for $\left|G H Z_{N}^{R}\right\rangle$. The following example shows that one need not imply the other:

Example 3. Consider the three-qubit system $\mathcal{H}=\mathbb{C}^{2} \otimes$ $\mathbb{C}^{2} \otimes \mathbb{C}^{2}$ and the resource state

$$
|\Phi\rangle=\frac{1}{\sqrt{3}}(|000\rangle+|110\rangle+|201\rangle) \in \mathbb{C}^{3} \otimes \mathbb{C}^{2} \otimes \mathbb{C}^{2}
$$

Then $|\Phi\rangle$ is universal for the problem of unambiguous discrimination in $\mathcal{H}$ but not perfect state discrimination.

In order to be universal for unambiguous state discrimination in $\mathcal{H}$, we need only show that $|\Phi\rangle$ can be transformed into both a $W$ state and a GHZ state. We can transform one state into another if they are related by a product matrix, and it is not hard to see that

$$
\begin{aligned}
|W\rangle & =\frac{1}{\sqrt{3}}(|100\rangle+|010\rangle+|001\rangle) \\
& =\left(\left[\begin{array}{lll}
0 & 1 & 1 \\
1 & 0 & 0
\end{array}\right] \otimes \mathcal{I}_{2} \otimes \mathcal{I}_{2}\right)|\Phi\rangle \\
|G H Z\rangle & =\frac{1}{\sqrt{2}}(|000\rangle+|111\rangle) \\
& =\sqrt{\frac{3}{2}}\left(\left[\begin{array}{lll}
0 & 1 & 1 \\
1 & 0 & 0
\end{array}\right] \otimes \sigma_{\mathrm{x}} \otimes \mathcal{I}_{2}\right)|\Phi\rangle
\end{aligned}
$$

This means that $|\Phi\rangle$ can be transformed into $|W\rangle$ and $|G H Z\rangle$. These are the two maximally entangled threequbit SLOCC classes, and Corollary 3 tells us that this is sufficient for $|\Phi\rangle$ to be universal for unambiguous discrimination.

On the other hand, if $\mathcal{B}$ is a basis of $\mathcal{H}$ which consists entirely of $W$ states and GHZ states (including at least one $G H Z$ state), then the set $|\Phi\rangle \otimes \mathcal{B}$ cannot be perfectly distinguished with LOCC. This can be seen by looking at the bipartite entanglement across the $A B: C$ split. Split this way, $|W\rangle$ and $|\Phi\rangle$ are in the same bipartite SLOCC class of $\mathbb{C}^{6} \otimes \mathbb{C}^{2}$, and they possess $H\left(\frac{2}{3}\right)<1$ units of bipartite entanglement, while the GHZ state has a full unit $H\left(\frac{1}{2}\right)=1$ of $A B: C$ entanglement.

Since average bipartite entanglement cannot increase under LOCC, there does not exist an LOCC protocol which transforms $|\Phi\rangle$ into each element of $\mathcal{B}$ with probability $\frac{1}{8}$; any protocol which sometimes gains entanglement by transforming $|\Phi\rangle$ into $|G H Z\rangle$ must also sometimes lose entanglement as well. This result implies that the elements of $|\Phi\rangle \otimes \mathcal{B}$ are not perfectly distinguishable with LOCC, according to Corollary 1 .

\section{CONCLUSION}

We have shown that for a fixed multipartite system there is often no resource from the same state space that can enable all complete orthogonal measurements on the whole system by LOCC. This is always the case when the dimensions of the $N \geq 3$ subsystems are all equal. This is in sharp contrast to the bipartite scenario, where a maximally entangled state of full Schmidt rank serves as a universal resource state. Furthermore, there exist orthonormal bases for which one cannot find any resource state from the same state space that perfectly distinguishes the basis states. This property of multipartite spaces is found to be typical even if we allow the dimensions of the subsystems to be different from one another; exceptions arise in scenarios where dimension of one of the subsystems is much larger than the dimensions of the other subsystems.

This line of questioning suggests many open problems. There is much that is not known about multipartite entanglement and the structure of SLOCC entanglement classes; and trying to understand the nature of universal resource states gives a possible line of approach. Certainly, it would be useful to find a complete characterization of universal resource states, perhaps finding ways to adapt the methods in [47] to do so. Even just bounding the necessary dimensions for such states would be a step in the right direction. The strength of Theorem 1 makes it seem like the search for universal states for unambiguous local discrimination would be the most promising. One could also try to determine whether the generalized GHZ states in Example 2 are universal for perfect state discrimination. This is related to the question of finding optimal resources in [36]; or even knowing whether a unique optimal resource exists. A larger question would be to find efficient universal resources to accomplish any quantum operation as efficiently locally as one could globally. For this, a pair of bell states $\left|\Phi_{\text {Bell }}^{N}\right\rangle$ could suffice (one to teleport everything into one place, and one to teleport them back); but it would be nice to have a smaller resource for this. It is hoped that this line of questioning will enable the continued exploration of the interplay between locality and entanglement.

SB is supported in part by DST-SERB project SR/S2/LOP-18/2012. SH is supported by a fellowship from CSIR, Govt. of India. MN acknowledges the continued support of the Office of Faculty Development at Saint Mary's College.

* Electronic address: som@jcbose.ac.in

† Electronic address: man6@stmarys-ca.edu

[1] R. Horodecki, P. Horodecki, M. Horodecki, and K. 
Horodecki, Quantum Entanglement, Rev. Mod. Phys. 81, 865 (2009).

[2] D. W. Berry, Implementation of multipartite unitary operations with limited resources", Phys. Rev. A 75, 032349 (2007).

[3] J. I. Cirac, W. Dür, B. Kraus, and M. Lewenstein, Entangling operations and their implementation using a small amount of entanglement", Phys. Rev. Lett. 86, 544 (2001).

[4] D. Collins, N. Linden, S. Popescu, Nonlocal content of quantum operations", Phys. Rev. A 64, 032302 (2001).

[5] S. Bandyopadhyay, G. Brassard, S. Kimmel, W. K. Wootters, Entanglement Cost of Nonlocal Measurements, Phys. Rev. A 80, 012313 (2009).

[6] S. Bandyopadhyay, R. Rahaman, W. K. Wootters, Entanglement cost of two-qubit orthogonal measurements, J. Phys. A: Math. Theor. 43, 455303 (2010).

[7] S. M. Cohen, Understanding entanglement as resource: Locally distinguishing unextendible product bases", Phys. Rev. A 77, 012304 (2008).

[8] C. H. Bennett, G. Brassard, C. Crépeau, R. Jozsa, A. Peres, and W. K. Wootters, Teleporting an unknown quantum state via dual classical and Einstein-Podolsky-Rosen channels, Phys. Rev. Lett. 70, 1895 (1993).

[9] C. H. Bennett and S. J. Wiesner, Communication via oneand two-particle operators on Einstein-Podolsky-Rosen states, Phys. Rev. Lett. 69, 2881 (1992).

[10] D. Jonathan and M. B. Plenio, Entanglement-assisted local manipulation of pure quantum states, Phys. Rev. Lett. 83, 3566 (1999).

[11] W. Dür, G. Vidal, J. I. Cirac, Three qubits can be entangled in two inequivalent ways, Phys. Rev. A 62, 062314 (2000).

[12] J. Walgate, A. J. Short, L. Hardy, and V. Vedral, Local distinguishability of multipartite orthogonal quantum states, Phys. Rev. Lett. 85, 4972 (2000).

[13] J. Walgate and L. Hardy, Nonlocality, Asymmetry and Distinguishing Bipartite States, Phys. Rev. Lett. 89, 147901 (2002).

[14] S. Ghosh, G. Kar, A. Roy, A. Sen (De), and U. Sen, Distinguishability of Bell states, Phys. Rev. Lett. 87, 277902 (2001).

[15] S. Ghosh, G. Kar, A. Roy, D. Sarkar, A. Sen (De), and U. Sen, Local indistinguishability of orthogonal pure states by using a bound on distillable entanglement, Phys. Rev. A 65, 062307 (2002).

[16] N. Yu, R. Duan, and M. Ying, Four Locally Indistinguishable Ququad-Ququad Orthogonal Maximally Entangled States, Phys. Rev. Lett. 109, 020506 (2012).

[17] S. Ghosh, G. Kar, A. Roy, and D. Sarkar, Distinguishability of maximally entangled states, Phys. Rev. A 70, 022304 (2004).

[18] H. Fan, Distinguishability and indistinguishability by local operations and classical communication, Phys. Rev. Lett. 92, 177905 (2004).

[19] M. Nathanson, Distinguishing bipartite orthogonal states by LOCC: best and worst cases, Journal of Mathematical Physics 46, 062103 (2005).

[20] J. Watrous, Bipartite subspaces having no bases distinguishable by local operations and classical communication, Phys. Rev. Lett. 95, 080505 (2005).

[21] R. Y. Duan, Y. Feng, Z. F. Ji, and M. S. Ying, Distinguishing arbitrary multipartite basis unambiguously using local operations and classical communication, Phys. Rev. Lett. 98, 230502 (2007).

[22] R. Y. Duan, Y. Feng, Y. Xin, and M. S. Ying, Distinguisha- bility of quantum states by separable operations, IEEE Trans. Inform. Theory 55, 1320 (2009).

[23] S. Bandyopadhyay, S. Ghosh and G. Kar, LOCC distinguishability of unilaterally transformable quantum states, New J. Phys. 13, 123013 (2011).

[24] M. Horodecki, A. Sen(De), U. Sen, and K. Horodecki, Local indistinguishability: more nonlocality with less entanglement, Phys. Rev. Lett. 90, 047902 (2003).

[25] A. Peres and W. K. Wootters, Optimal Detection of Quantum Information, Phys. Rev. Lett. 66, 1119 (1991); S. Massar and S. Popescu, Optimal extraction of information from finite quantum ensembles, Phys. Rev. Lett. 74, 1259 (1995).

[26] C. H. Bennett, D. P. DiVincenzo, C. A. Fuchs, T. Mor, E. Rains, P. W. Shor, J. A. Smolin, and W. K. Wootters, Quantum Nonlocality without Entanglement, Phys. Rev. A 59, 1070 (1999).

[27] C. H. Bennett, D. P. DiVincenzo, T. Mor, P. W. Shor, J. A. Smolin, and B. M. Terhal, Unextendible Product Bases and Bound Entanglement, Phys. Rev. Lett. 82, 5385 (1999); D. P. DiVincenzo, T. Mor, P. W. Shor, J. A. Smolin, B. M. Terhal, Unextendible Product Bases, Uncompletable Product Bases and Bound Entanglement, Comm. Math. Phys. 238, 379 (2003).

[28] J. Calsamiglia, J. I. de Vicente, R. Munoz-Tapia, E. Bagan, Local discrimination of mixed states, Phys. Rev. Lett. 105, 080504 (2010).

[29] S. Bandyopadhyay, More nonlocality with less purity, Phys. Rev. Lett. 106, 210402 (2011).

[30] S. Bandyopadhyay, A. Cosentino, N. Johnston, V. Russo, J. Watrous, and N. Yu, Limitations on separable measurements by convex optimization," IEEE Transactions on Information Theory, Vol 61, Issue 6, Pages: 3593-3604 (2015).

[31] S. Virmani, M. F. Sacchi, M. B. Plenio, D. Markham, Optimal local discrimination of two multipartite pure states, Phys. Lett. A. 288 (2001).

[32] B. M. Terhal, D. P. DiVincenzo, and D. W. Leung, Hiding bits in Bell states, Phys. Rev. Lett. 86, 5807 (2001).

[33] D. P. DiVincenzo, D. W. Leung, and B. M. Terhal, Quantum data hiding, IEEE Trans. Inf. Theory 48, 580 (2002).

[34] T. Eggeling, and R. F. Werner, Hiding classical data in multipartite quantum states, Phys. Rev. Lett. 89, 097905 (2002).

[35] W. Matthews, S. Wehner, A. Winter, Distinguishability of quantum states under restricted families of measurements with an application to quantum data hiding, Comm. Math. Phys. 291, Number 3 (2009).

[36] C. Guo, E. Chitambar, and R. Duan, Common Resource State for Preparing Multipartite Quantum Systems via Local Operations and Classical Communication, arXiv:1601.06220 [quant-ph] (2016).

[37] D. Markham and B. C. Sanders, Graph states for quantum secret sharing, Phys. Rev. A 78, 042309 (2008).

[38] A. Chefles, Unambiguous discrimination between linearly independent quantum states, Phys.Lett. A 239 (1998).

[39] G. Gour and N. R. Wallach. Classification of Multipartite Entanglement of All Finite Dimensionality. Phys. Rev. Lett. 111060502 (2013).

[40] A. Chefles, Condition for unambiguous state discrimination using local operations and classical communication, Phys. Rev. A 69050307 (2004).

[41] M. Hebenstreit, C. Spee, and B. Kraus, The MES of tripartite qutrit states and pure state separable transformations 
which are not possible via LOCC, Phys. Rev. A 93, 012339 (2016).

[42] J. Eisert and H.J. Briegel, The Schmidt Measure as a Tool for Quantifying Multi-Particle Entanglement, Phys. Rev. A 64 (2001).

[43] M. Hein, J. Eisert and H.J. Briegel, Multi-party entanglement in graph states. Phys. Rev. A 69, 062311 (2004).

[44] M. Hajdušek and M. Murao, Direct evaluation of pure graph state entanglement, New Journal of Physics 15 013039 (2013).
[45] L. Chen, E. Chitambar, R. Duan, Z. Ji, and A. Winter, Tensor Rank and Stochastic Entanglement Catalysis for Multipartite Pure States, Phys. Rev. Lett. 105, 200501 (2010).

[46] N. Yu, E. Chitambar, C. Guo, and R. Duan, The Tensor Rank of the Tripartite State $|W\rangle^{\otimes n}$, Phys. Rev. A 81, 014301 (2010).

[47] G. Gour and N. R. Wallach, Necessary and sufficient conditions for local manipulation of multipartite pure quantum states, New J. Phys. 13073013 (2011). 